\title{
Thermococcus camini sp. nov., a hyperthermophilic and piezophilic archaeon isolated from a deep-sea hydrothermal vent at the Mid-Atlantic Ridge
}

\author{
Damien Courtine ${ }^{1,2,3}$, Erwann Vince ${ }^{1,2}$, Loïs Maignien ${ }^{1,2}$, Xavier Philippon $^{1,2}$, Nicolas Gayet ${ }^{4}$, Zongze Shao ${ }^{2,5}$ and \\ Karine Alain ${ }^{1,2, *}$
}

\begin{abstract}
A coccoid-shaped, strictly anaerobic, hyperthermophilic and piezophilic organoheterotrophic archaeon, strain Iri35c ${ }^{\top}$, was isolated from a hydrothermal chimney rock sample collected at a depth of $2300 \mathrm{~m}$ at the Mid-Atlantic Ridge (Rainbow vent field). Cells of strain Iri35 $\mathrm{c}^{\top}$ grew at $\mathrm{NaCl}$ concentrations ranging from 1-5\% (w/v) (optimum 2.0\%), from pH 5.0 to 9.0 (optimum 7.0-7.5), at temperatures between 50 and $90^{\circ} \mathrm{C}$ (optimum $75-80^{\circ} \mathrm{C}$ ) and at pressures from 0.1 to at least $50 \mathrm{MPa}$ (optimum: 10-30 MPa). The novel isolate grew on complex organic substrates, such as yeast extract, tryptone, peptone or beef extract, preferentially in the presence of elemental sulphur or L-cystine; however, these molecules were not necessary for growth. Its genomic DNA G+C content was $54.63 \mathrm{~mol} \%$. The genome has been annotated and the metabolic predictions are in accordance with the metabolic characteristics of the strain and of Thermococcales in general. Phylogenetic analyses based on $16 \mathrm{~S}$ rRNA gene sequences and concatenated ribosomal protein sequences showed that strain Iri $35 c^{\top}$ belongs to the genus Thermococcus, and is closer to the species T. celericrescens and T. siculi. Average nucleotide identity scores and in silico DNA-DNA hybridization values between the genome of strain $\mid r i 35 c^{\top}$ and the genomes of the type species of the genus Thermococcus were below the species delineation threshold. Therefore, and considering the phenotypic data presented, strain Iri35c ${ }^{\top}$ is suggested to represent a novel species, for which the name Thermococcus camini sp. nov. is proposed, with the type strain Iri35 $\mathrm{c}^{\top}$ $\left(=U B O C C M-2026^{\top}=\right.$ DSM 111003').
\end{abstract}

Contrary to many orders within the Archaea, the order Thermococcales has led to the isolation of numerous cultured representatives that have been the subject of physiological and genomic studies. Thermococcales populate a variety of high-temperature natural ecosystems (deep-sea and shallowmarine hydrothermal vents, terrestrial hot springs, oil reservoirs, solfataric systems, etc.), the most important of which are marine hydrothermal vents, from which the largest number of isolates originate [1]. Among the three genera (Thermococcus, Pyrococcus and Palaeococcus) of the order Thermococcales [2], it is the genus Thermococcus that has led to the isolation of the largest number of species, with 33 species (with names validly recognized by the ICSP, the International Committee on Systematics of Prokaryotes) recorded to date. Physiological studies of these species have shown that this genus is composed of hyperthermophilic anaerobic taxa developing mainly through chemoorganoheterotrophy by coupling the oxidation of peptides or sugars to the reduction of elemental sulphur and protons [2]. This genus is also described to contain carboxydotrophic species (i.e. $[3,4]$. Thanks to

Author affiliations: 'Univ Brest, CNRS, Ifremer, Laboratoire de Microbiologie des Environnements Extrêmes LM2E, IUEM, Rue Dumont d'Urville, F-29280 Plouzané, France; ${ }^{2}$ IRP 1211 MicrobSea, Sino-French Laboratory of Deep-Sea Microbiology, LM2E (Plouzané, France)-KLAMBR, Xiamen, PR China; ${ }^{3}$ Aix Marseille Univ, CNRS, INSERM, CIML, Turing Centre for Living Systems, Marseille, France; ${ }^{4}$ fremer, EEP, F-29280 Plouzané, France; ${ }^{5}$ Key Laboratory of Marine Genetic Resources, Third Institute of Oceanography, Ministry of Natural Resources, Xiamen 361005, PR China.

*Correspondence: Karine Alain, karine.alain@univ-brest.fr

Keywords: Thermococcus; hydrothermal; hyperthermophilic; piezophilic.

Abbreviations: ANI, average nucleotide identity; CAPSO, N-cyclohexyl-3-aminopropanesulfonic acid; DMSO, dimethylsulfoxide; HEPES,

4-(2-hydroxyethyl)-1-piperazineethanesulfonic acid; HOMOPIPES, Homopiperazine-1,4-bis(2-ethanesulfonic acid); ICSP, International Committee on Systematics of Prokaryotes; MAR, mid-atlantic ridge; MES, 2-(N-morpholino)ethanesulfonic acid; PIPES, piperazine-N,N'-bis(2-ethanesulfonic acid); TAPS, N-[Tris(hydroxymethyl)methyl]-3-aminopropanesulfonic acid, [(2-Hydroxy-1,1-bis(hydroxymethyl)ethyl)amino]-1-propanesulfonic acid; UBOCC, University of Brest (UBO) Culture Collection.

The DDBJ[/GenBank/ENA] accession number for the genome sequence of Thermococcus camini Iri35c ${ }^{\top}$ sp. nov. is LR881183.1. The complete 16S rRNA gene sequence of T. camini sp. nov. strain Iri35 $\mathrm{c}^{\top}$ is available at GenBank/EMBL/DDBJ/PIR under accession no. MT921160.

One supplementary table and two supplementary figures are available with the online version of this article. 
the different genetic tools available to work on models of Thermococcus species, such as Thermococcus kodakarensis or Thermococcus barophilus for example, many functional studies have been carried out and have allowed significant advances in our knowledge of their metabolism, genomic maintenance mechanisms and biological adaptations, instrumental in advancing our understanding of the biology of the Thermococcales and of the Archaea in general (e.g. $[5,6]$. In addition to being important and ubiquitous players in the hot areas of the hydrothermal ecosystems, Thermococcus species are also of particular interest for learning more about the cellular processes at the limits of life, as this genus contains extremophilic and polyextremophilic organisms, adapted to one or more extreme physical or chemical conditions of their natural habitat. In this way, all taxa of the genus Thermococcus are hyperthermophilic, capable for some of them to divide up to a maximal temperature of $100^{\circ} \mathrm{C}$ (e.g. T. eurythermalis, T. kodakarensis, T. peptonophilus) [7-9], tolerating in addition high doses of gamma radiation for some of them (e.g. T. gammatolerans resist up to $30 \mathrm{kGy}$ of $\gamma$-radiation) [10], and with better growth under high hydrostatic pressure (e.g. T. barophilus, T. piezophilus; T. piezophilus holds the current record of pressure range for growth, growing from atmospheric pressure to $130 \mathrm{MPa})[11,12]$ or under alkaline $\mathrm{pH}$ conditions for others (e.g. T. alcaliphilus) [13].

In this article, we describe a novel hyperthermophilic organoheterotrophic sulphur-reducer, strain Iri35 $c^{\mathrm{T}}$, isolated from a hydrothermal rock sample from the Rainbow vent field, at the Mid-Atlantic Ridge. Genotypic and phenotypic characteristics meet the standard nomenclatural criteria to delineate a novel species. We propose to name this new species Thermoccocus camini.

A chimney rock sample was collected at a depth of $2300 \mathrm{~m}$ from a hydrothermal vent at the Rainbow vent field $\left(36^{\circ} 13^{\prime} \mathrm{N}\right.$, $33^{\circ} 54^{\prime} \mathrm{W}$ ), at the Mid-Atlantic Ridge, in June 2001, during the IRIS oceanographic cruise. Onboard, the sample was preserved in a sealed sterile anoxic vial and stored at $4{ }^{\circ} \mathrm{C}$, since the objective was to cultivate (hyper)thermophiles from this sample. Once in the lab, enrichment cultures followed by three series of dilutions-to-extinction were performed at $85^{\circ} \mathrm{C}$, in reduced TRM medium ( $\mathrm{pH} 6.8$ ), containing $5 \mathrm{~g} \mathrm{l}^{-1}$ elemental sulphur, as described elsewhere [14]. A collection of pure strains was then deposited in the UBOCC collection (https://ent.univ-brest.fr/lm2e/home/\#/), at $-80^{\circ} \mathrm{C}$ with $5 \%(\mathrm{v} / \mathrm{v})$ DMSO. The strain Iri35 $\mathrm{c}^{\mathrm{T}}$ described here bears the accession number UBOCC-M-2026 ${ }^{\mathrm{T}}$. The purity of this isolate was confirmed routinely by microscopic examination, and by sequencing of its genome.

The genomic DNA of Iri35 $c^{\mathrm{T}}$ was extracted using a phenolchloroform procedure. The TruSeq DNA PCR-free kit (Illumina, USA) was then used to prepare paired-end sequencing libraries with an average insert size of $550 \mathrm{nt}$, before the genome was sequenced using the Illumina's MiSeq technology ( $2 \times 300$ bp paired-reads, V3 chemistry), at the Marine Biological Laboratory (Woods Hole, MA, USA). Paired-end reads were filtered with the Python package illumina-utils using the command 'iu-filter-quality-minoche' and default parameters $[15,16]$. The de novo assembly of the genome was carried out using CLC Genomics Workbench v8.5.1 (https:// www.qiagenbioinformatics.com/products/clc-genomicsworkbench). A total of 482309 read pairs of $300 \mathrm{bp}$ were used for the genome assembly, representing a mean coverage of about 120x. The assembled genome was analysed and annotated with the MicroScope Microbial Genome Annotation and Analysis Platform (MaGe) (https://mage.genoscope.cns. fr/microscope/home/index.php) using KEGG and BioCyc databases [17]. It consists of one circular chromosome of 2022529 base pairs in size, and has a G+C content of 54.63 $\mathrm{mol} \%$. CheckM estimated the genome to be $100 \%$ complete based on the presence of default single-copy marker genes (four markers were missing) and without any contamination. The genome consists of 2204 encoding protein sequences, 46 tRNA genes, a single 16S-23S rRNA operon, two $5 \mathrm{~S}$ rRNA, and 14 miscellaneous RNA genes. This genome is available in DDBJ/ENA/GenBank under the accession number LR881183.1 (BioProject: PRJEB40155).

Pairwise 16S rRNA gene sequence similarity was determined using the EzTaxon-e server (http://eztaxon-e.ezbiocloud. net/ [18]). The $16 \mathrm{~S}$ rRNA gene sequences were aligned using MAFFT v7.427 (parameters -maxiterate 1000 -localpair) [19], and the alignment was trimmed with BMGE v1.12 (default parameters) [20]. Then, PhyML v. 3.3.20190909 was used to build the tree thanks to the web-server http://www.atgc-montpellier.fr/phyml/ [21]. The evolutionary model was selected with the SMS algorithm [22] and the branch support was computed with the aLRT SH-like method. The tree was visualized with iToL [23] and rooted between the Thermococcus and Pyrococcus genera. Since the $16 \mathrm{~S}$ rRNA gene sequence is highly conserved and therefore not very discriminating between Thermoccocales, a phylogenetic tree based on ribosomal proteins was also constructed. This phylogenomic tree was based on the concatenation of 49 ribosomal proteins shared by all genomes. Each protein was aligned and trimmed separately with MAFFT (parameters -globalpair -maxiterate 1000) and BMGE (default parameters), respectively. Then, each alignment block was concatenated into a single alignment that was submitted to PhyML. The evolutionary model was selected with SMS and the branch support was computed with the aLTR $\mathrm{SH}$-like method. The tree was visualized with iToL and rooted between the Thermococcus and Pyrococcus branches. Average Nucleotide Identity scores (ANI) were calculated using two methods: OrthoANIu from the EzBioCloud web server (https://www.ezbiocloud.net/tools/ani) [24]; and ANIb values by JSpeciesWS Online Service ([25]; http://jspecies.ribohost. com/jspeciesws/\#analyse). These ANI scores were calculated between the genome of strain Iri35c $c^{\mathrm{T}}$ and the genomes of the four closest type species whose genomes are available: T. celericrescens TS2 ${ }^{\mathrm{T}}$ (NZ_LLYW00000000.1), T. siculi RG20 ${ }^{\mathrm{T}}$ (NZ_CP015103.1), T. cleftensis CL1 ${ }^{\mathrm{T}}$ (NC_018015.1) and T. pacificus P-4 ${ }^{\mathrm{T}}$ (NZ_CP015102.1). Digital DNA-DNA hybridization $(\mathrm{dDDH})$ scores were also determined by the genome-to-genome distance calculator (GGDC 2.1), using the formula 2 [26]. 


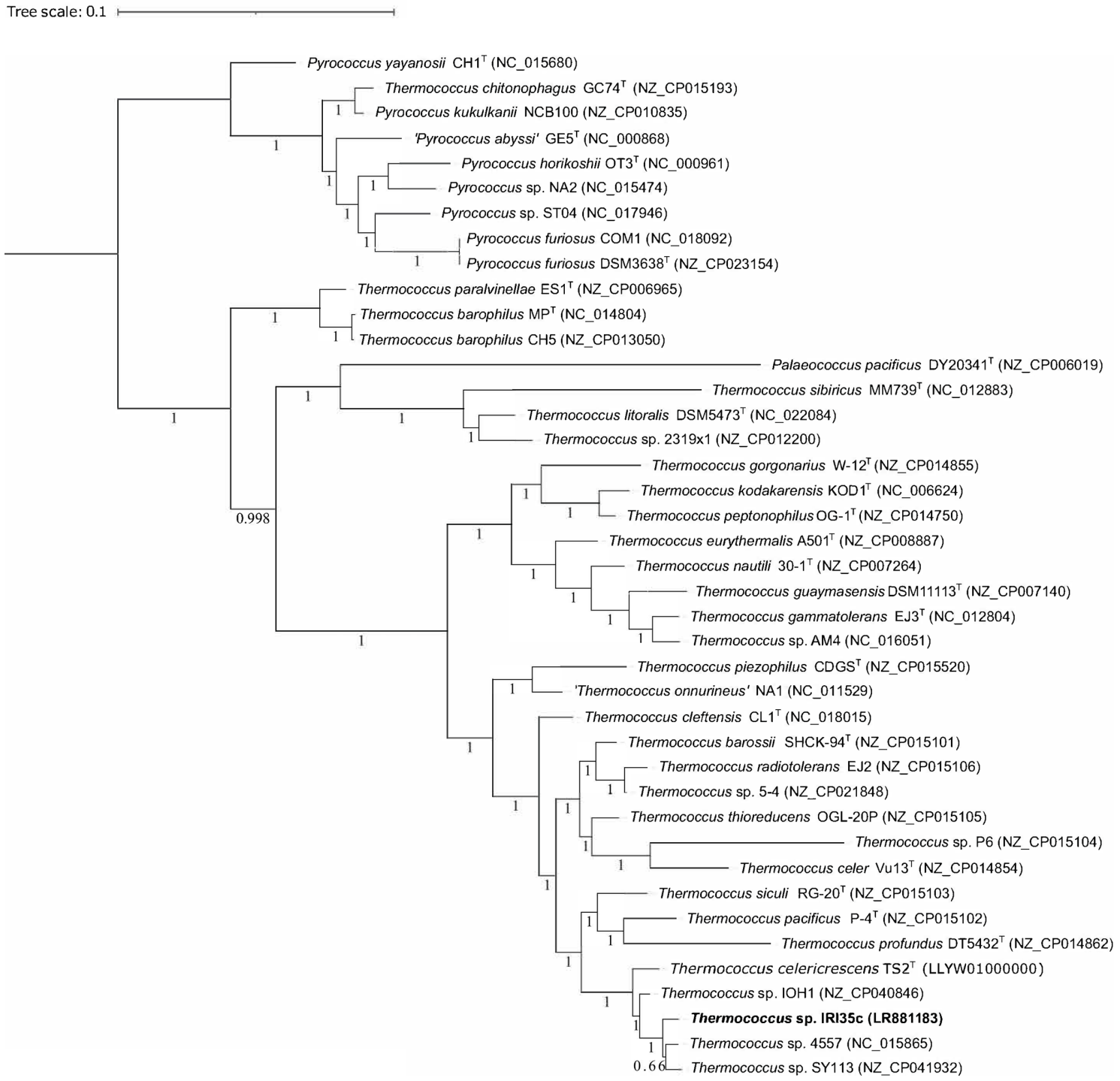

Fig. 1. Phylogenetic tree of the strain Iri35 $\mathrm{c}^{\top}$ and representatives of Thermococcales, based on 49 ribosomal proteins (Proteins associated with the large ribosomal subunit: L1, L2, L3, L4, L6, L7AE, L11, P1 (=L12P), L13, L15, L15E, L18, L18A, L18E, L19E, L21E, L22, L24, L29, L30, L30E, L31E, L32E, L37AE, L37E, L39E, L40E, L44E; Proteins associated with the small ribosomal subunit: S2, S3, S3AE, S4, S4E, S5, S6E, S7, S8, S8E, S9, S10, S12, S13, S15, S17, S17E, S19, S19E, S27E, S28E). The tree was built by maximum-likelihood (PhyML). Branch support, shown on the tree, was computed with the aLTR SH-like method. Bar, 0.1 amino-acid substitution rate.

Phylogenetic analyses of the 16S rRNA gene sequences and concatenated ribosomal proteins both confirmed that the new isolate branched within the archaeal genus Thermococcus (Figs 1 and S1, available in the online version of this article). The most closely related species of strain Iri35 $\mathrm{c}^{\mathrm{T}}$ were Thermococcus celericrescens $\mathrm{TS}^{\mathrm{T}}$ (99.66\% 16S rRNA gene sequence similarity), T. siculi RG-20 $0^{\mathrm{T}}$ (99.26\%), T. barossii SHCK-94 ${ }^{\mathrm{T}}$ (98.79\%), T. thioreducens DSM $14981^{\mathrm{T}}$ (98.79\%), T. hydrothermalis AL662 ${ }^{\mathrm{T}}$ (98.75\%), T. cleftensis CL1 ${ }^{\mathrm{T}}(98.59 \%)$ and T. pacificus $\mathrm{P}-4^{\mathrm{T}}(98.05 \%)$. Due to the high degree of similarity between the sequences of $16 \mathrm{~S}$ rRNA genes, overall genomes relatedness indices were calculated. The genomes of strain Iri35 $\mathrm{c}^{\mathrm{T}}$ and of its closest relatives shared OrthoANIu values ranging from $79.45-88.20 \%$ (Tables 1 and S1), and ANIb values between 78.68 and $87.31 \%$ (Table 1). These values are far below the ANI value of $95-96 \%$ generally accepted as a boundary for species delineation [27]. Digital DNA-DNA hybridization scores were also well below the DDH threshold level for species demarcation (70\%), with values from 22.20 $35.10 \%$ between the genome of strain Iri35 $\mathrm{c}^{\mathrm{T}}$ and the genomes of its closest neighbours, respectively (Tables 1 and S1) [28]. These results based on standard genomes relatedness indexes provide evidence that strain Iri35 $c^{\mathrm{T}}$ represents a new genomic species [29].

Morphological characteristics of strain Iri35c $c^{\mathrm{T}}$ were determined by light microscopy (Olympus BX60 and CX40) and scanning electron microscopy (FEI Quanta 200) (Fig. S2). 
Table 1. Characteristics differentiating strain Iri35c ${ }^{\top}$ from closest species of the genus Thermococcus

Strains: 1, Iri35c (data from this study); 2, T. celericrescens TS2 ${ }^{\top}$ [38]; 3, T. siculi RG20 ${ }^{\top}$ [39] ; 4, T. cleftensis CL1 ${ }^{\top}$ [40, 41]; 5, T. pacificus P-4 ${ }^{\top}$ [32]. Characteristics are scored as: +, positive ; -, negative; S, stimulatory; R, required; ND, not determined.

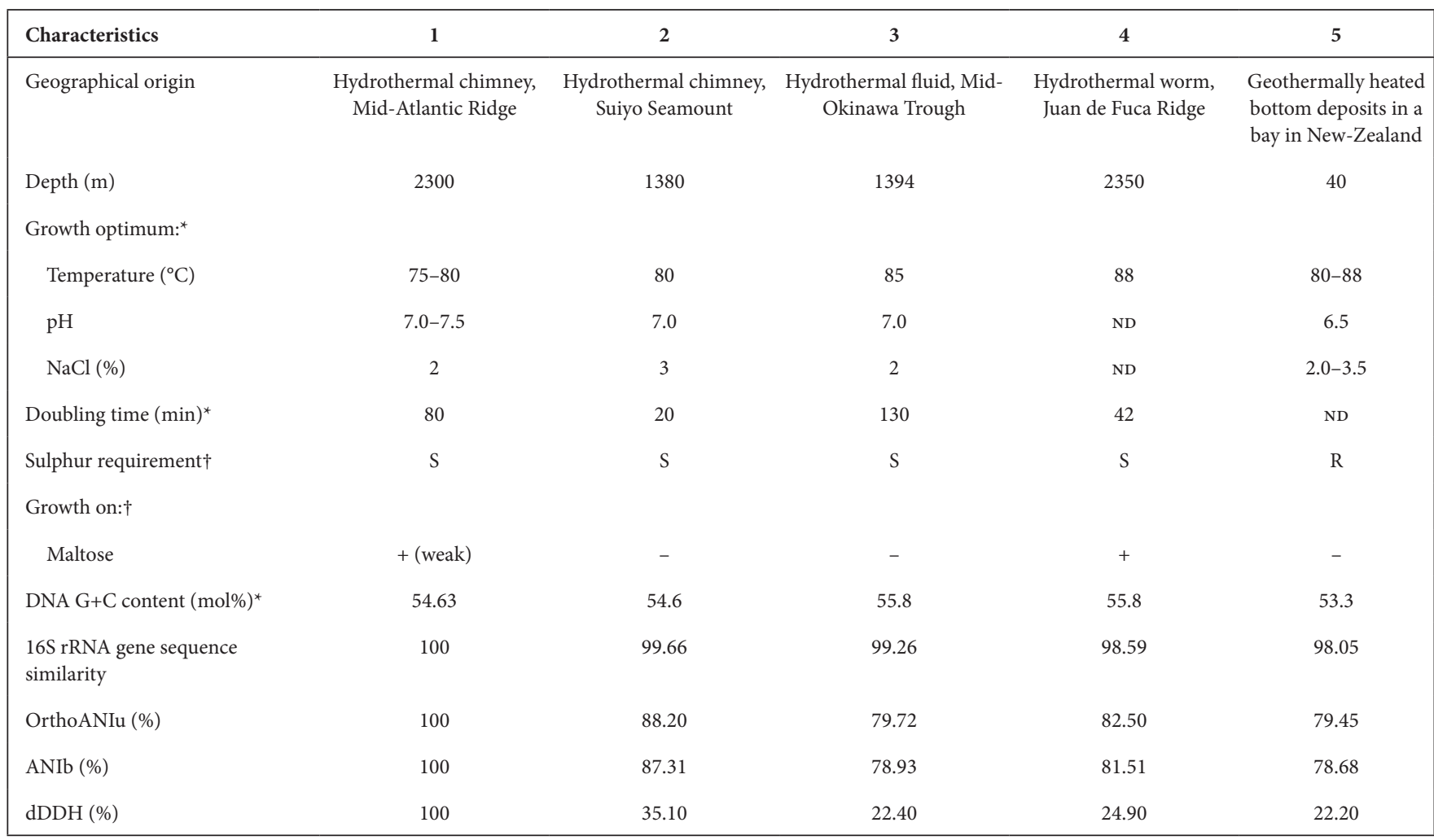

*Data from the literature.

†Data obtained for the five strains under same experimental laboratory conditions.

Cells were motile cocci that occurred generally singly and divided by constriction (Fig. S2). Under optimal growth conditions and in the mid-exponential phase of growth, cells occurred as irregular cocci of $0.8-1.7 \mu \mathrm{m}$ in diameter (mean $1.1 \pm 0.2 \mu \mathrm{m}, n=31)$.

Unless stated otherwise, physiological assays were carried out anaerobically $\left(\mathrm{N}_{2}\right.$ headspace) in modified Ravot medium, at $80^{\circ} \mathrm{C}$, in duplicates, in the presence of elemental sulphur, as described elsewhere [11]. Growth tests were generally carried out as described previously [11]. Cells were routinely counted by direct cell counting using a modified Thoma chamber (depth $10 \mu \mathrm{m}$ ), and checked by flow cytometry (CyFlowSpace, Sysmex Partec, GmbH, Görlitz, Germany). Cells were fixed with $2.5 \%(\mathrm{v} / \mathrm{v})$ glutaraldehyde (Sigma) and stored at $-80^{\circ} \mathrm{C}$, before counting by the two methods described above. Determination of the temperature range for growth was carried out at $45,50,55,60,65,70,75,80,85,90,95$ and $100^{\circ} \mathrm{C}$. The isolate was hyperthermophilic and grew between 50 and $90^{\circ} \mathrm{C}$ with an optimum at $75-80^{\circ} \mathrm{C}$. Salt tolerance was tested at $80^{\circ} \mathrm{C}$ with various concentrations of $\mathrm{NaCl}(0,1,2$, $3,4,5,6,7$ and $8 \%, w / v)$. Strain Iri35 $\mathrm{c}^{\mathrm{T}}$ required $\mathrm{NaCl}$ for growth and grew at $\mathrm{NaCl}$ concentrations between 1 and $5 \%$ (optimum: 2\%). The $\mathrm{pH}$ range for growth was tested from $\mathrm{pH}$ 3.0 to $\mathrm{pH} 10.0$ (initial $\mathrm{pH}$ at $20^{\circ} \mathrm{C}$ ) with increments to one unit near the limits of the $\mathrm{pH}$ range, and with increments of 0.5 unit around the optimum. For this experiment, we used the following buffers (each at $20 \mathrm{mM}$, Sigma-Aldrich): for $\mathrm{pH}$ 3.0, no buffer; for $\mathrm{pH} 4.0$ and 5.0, HOMOPIPES buffer; for $\mathrm{pH}$ 5.5-6.5, MES buffer; for $\mathrm{pH}$ 7.0, PIPES buffer; for pH 7.5-8.0, HEPES buffer; for $\mathrm{pH} 8.5$, TAPS buffer; for $\mathrm{pH}$ 9.0 and 10.0, CAPSO buffer. Growth of strain Iri35c $c^{\mathrm{T}}$ was observed from $\mathrm{pH} 5.0$ to $\mathrm{pH}$ 9.0, with an optimum around 7.0-7.5. The pressure range for growth of strain Iri $35 c^{\mathrm{T}}$ was tested into high-pressure high-temperature reactors (Top Industrie, Vaux-le-Pénil, France), at 0.1, 10, 20, 30, 40 and $50 \mathrm{MPa}$, as described previously [30]. The novel isolate was piezophilic, growing from atmospheric pressure $(0.1 \mathrm{MPa})$, to at least $50 \mathrm{MPa}$, and showed optimal growth at $10-30 \mathrm{MPa}$. Under optimal growth conditions $\left(80^{\circ} \mathrm{C}, \mathrm{pH} 7.0,2 \% \mathrm{NaCl}\right.$, $5 \mathrm{gl}^{-1} \mathrm{~S}^{\circ}$, and $20 \mathrm{MPa}$ ), the doubling time of the novel isolate was $80 \mathrm{~min}$.

Utilization of various individual substrates for growth was tested in a basal medium supplemented with $0.05 \%(\mathrm{w} / \mathrm{v})$ yeast extract (YE) as growth factor, and without this growth factor, as described previously [11]. The following substrates were tested, at the final concentrations shown in brackets: tryptone $(0.5 \% \mathrm{w} / \mathrm{v})$, peptone $(0.5 \% \mathrm{w} / \mathrm{v})$, yeast extract $(0.5 \% \mathrm{w} / \mathrm{v})$, beef extract $(0.5 \% \mathrm{w} / \mathrm{v})$, casamino acids $(0.4 \% \mathrm{w} / \mathrm{v})$, casein 
$(0.5 \% \mathrm{w} / \mathrm{v})$, formate $(20 \mathrm{mM})$, acetate $(20 \mathrm{mM})$, pyruvate $(20 \mathrm{mM})$, fumarate $(20 \mathrm{mM})$, propionate $(20 \mathrm{mM})$, succinate $(20 \mathrm{mM})$, maltose $(20 \mathrm{mM})$, fructose $(20 \mathrm{mM})$, lactose $(20 \mathrm{mM})$, ribose $(20 \mathrm{mM})$, galactose $(20 \mathrm{mM})$ and glucose $(20 \mathrm{mM})$. Positive controls were performed for all tests, and unsupplemented media were used as negative controls. To examine the ability of the strain to grow in the absence of elemental sulphur, cells were cultivated in modified Ravot medium without sulphur. Alternative electron acceptors were also tested in a sulphur-depleted medium, under a gas phase of $\mathrm{N}_{2}(100 \%, 150 \mathrm{kPa})$ : L-cystine $\left(5 \mathrm{~g} \mathrm{l}^{-1}\right)$, polysulphides $(0.5 \mathrm{mM})$, thiosulphate $(20 \mathrm{mM})$, sulphate $(20 \mathrm{mM})$, sulphite $(5 \mathrm{mM})$, nitrate $(20 \mathrm{mM})$, nitrite $(5 \mathrm{mM})$ and dioxygen $(0.5$, $5,20 \% \mathrm{v} / \mathrm{v})$. Growth was monitored over 3 days of incubation. The results were considered positive when growth was still observed after two successive subcultures $(1 / 100$ th subculturing), on the test medium. All tests were performed in duplicates and growth was confirmed after microscopic observation. Hydrogen sulphide production was monitored with a colorimetric test as described previously [31]. Gas concentrations in the headspace phase were determined using a modified INFICON/MicroGC FUSION Gas Analyser (INFICON, Basel, Switzerland) fitted with a pressure gauge (CTE8005AY0, Sensortechnics GmbH) and two conductivity detectors. Separation was performed using two columns: molecular sieve $10 \mathrm{~m}$ column and argon as a carrier gas; and a RT-Q12 m using helium as a carrier gas. Cations and anions produced from peptone and yeast extract fermentation were identified by ionic chromatography on a Dionex ICS-900 Ion Chromatography System (Dionex, Camberley UK) coupled with a CERS $5004 \mathrm{~mm}$ suppressor and a DS5 conductivity detector $\left(40^{\circ} \mathrm{C}\right)$ and fitted with a RFC-10 Reagent-Free Controller, an ASDV autosampler, and an IonPac CS16 column maintained at $60^{\circ} \mathrm{C}$ in a UltiMate 3000 Thermostated Column Compartment (Thermo Scientific, Waltham, MA, USA).

In the presence of elemental sulphur and under strict anaerobic conditions, complex carbon sources such as yeast extract, peptone, tryptone and beef extract supported fast and significant growth. Cations and anions produced by the fermentation of peptone and yeast extract included formate, acetate, propionate, isobutyrate, succinate or malate, isovalerate, ammonia, carbon dioxide and hydrogen sulphide. The presence of all or part of these organic acids has already been reported as products of amino acid catabolism in other Thermococcus species (T. gorgonarius, T. alcaliphilus, T. piezophilus...) $[11,13,32]$. The production of ammonium, already reported in T. alcaliphilus for example, probably results from the transamination or oxidation of amino acids [13]. In the absence of elemental sulphur, the strain produced hydrogen by fermentation of peptone and yeast extract. Under our experimental conditions, no obvious growth was observed with the other carbon sources tested, with the exception of maltose which slightly enhanced growth. Poor growth was observed on peptone and yeast extract under pure fermentation conditions, in the absence of sulphur species. Although not necessary for growth, L-cystine and elemental sulphur clearly stimulated the growth of the strain, and were both reduced to hydrogen sulphide. None of the other sulphur species tested (sulphate, thiosulphate, sulphite, polysulphides) had an effect on growth. Nitrate, nitrite and oxygen (aerobic to microaerophilic conditions) were not used by the cells as terminal electron acceptors. Growth by carboxydotrophy was not tested as the gene encoding the carbon monoxide dehydrogenase CooF, one central protein in the Thermococcales's carbon monoxide metabolism, was absent from the genome. Similarly, growth on chitin and starch has not been tested because the degradation pathways of these compounds are incomplete based on the MetaCyc database.

The annotation of the Iri35 $c^{\mathrm{T}}$ genome confirmed that the strain has the genetic potential to grow organoheterotrophically from peptides, amino acids and carbohydrates. Concerning the catabolism of peptides and amino acids, the genome encodes several proteases and two central enzymes involved in the oxidation of amino acids into their respective organic acids: the alanine aminotransferase (AlaAT; TIRI35C_0605) and the glutamate dehydrogenase (GDH; TIRI35C_0530). It has been proposed that these two enzymes, AlaAT and $\mathrm{GDH}$, may act in a coordinated manner to maintain the redox balance in Thermococcales metabolism and to form an electron sink, by modulating the transformation of pyruvate towards acetate or alanine; alanine could be accumulated as an end-product under high $\mathrm{H}_{2}$ partial pressure and in the absence of sulphur [33, 34]. Based on MicroCyc, the degradation pathways for six amino acids are complete in the genome of strain Iri $35 c^{\mathrm{T}}$ : alanine, arginine, asparagine, aspartate, glutamine and glycine. These results are congruent with the growths observed on complex proteinaceous substrates. With regard to the catabolism of sugars, the genome contains notably an $\mathrm{ABC}$-type maltose/maltodextrin transport system MalEFGK (TIRI35C_0120- TIRI35C_0122-0124), a cyclomaltodextrinase (TIRI35C_0125), three alpha-amylases (TIRI35C_1801; TIRI35C_1925; TIRI35C_2170), one pullullanase (TIRI35C_0121) and a complete EmbdenMeyerhof-Parnas pathway V (including three enzymes unique to this modified version of the glycolysis pathway, typically found within Thermococcales [35], namely: an ADP-dependent glucokinase (locus tag TIRI35C_0588), an ADP-dependent phosphofructokinase (TIRI35C_1487) and a glyceraldehyde-3-phosphate:ferredoxin oxidoreductase (TIRI35C_2006)). It encodes also full degradation pathways for glycerol, D-mannose and melibiose. In Thermococcales the catabolism of carbohydrates produces reducing equivalents as reduced ferredoxins, while the catabolism of amino acids produces both NADPH and reduced ferredoxins. Reduced ferredoxins are used by two main types of membrane-bound hydrogenases, Mbh and Mbs (previously termed Mbx), which conserve energy by creating an ion gradient across the membrane, and this gradient can then be used by an ATP synthase (TIRI35C_0071-0079) to produce energy [36]. These two main types of membrane-bound hydrogenases, which are respectively hydrogenogenic (Mbh) and sulphidogenic (Mbs) are mobilized according to whether or not there is sulphur in the culture medium [36]. Both are present in the 
genome of strain Iri35 ${ }^{\mathrm{T}}$ (Mbh: TIRI35C_2078-2097; Mbs: TIRI35C_0918-0921, TIRI35C_0406-0409). In addition to these membrane-bound hydrogenases, the genome encodes also cytosolic hydrogenases. The gene cluster encoding the formate hydrogenlyase complex which is present in several Thermococcales, combines $\mathrm{H}_{2}$ oxidation with $\mathrm{CO}_{2}$ reduction to form formate (which could mitigate $\mathrm{H}_{2}$ saturation under hydrogenogenic growth conditions) [37], is incomplete in this draft genome, which suggests that formate is produced by another pathway in this strain. However, the genome codes for the pyruvate formate lyase activating-enzyme (TIRI35C_0782) which allows the conversion of pyruvate (the end-product of glycolysis) into formate and acetyl Co-A, and might explain the formate production as a catabolic end-product.

In summary, like many Thermococcales, strain Iri35 $c^{\mathrm{T}}$ is a strict anaerobic archaeon growing chemoorganoheterotrophically on complex proteinaceous substrates, whose growth is very largely stimulated by the presence of elemental sulphur or L-cystine to detoxify the dihydrogen produced by its metabolism (deleterious to its growth) and thus produce a high biomass in culture.

As is very often observed in the genus Thermococcus, which forms a fairly homogeneous group in terms of physiology, there are very few phenotypic differences between strain Iri35 $\mathrm{c}^{\mathrm{T}}$ and its phylogenetically closely related species. These differences are summarized in Table 1 . Strain $\operatorname{Iri} 35 c^{\mathrm{T}}$ is distinguishable from $T$. pacificus by the fact that it does not require the presence of sulphur to grow. In addition, the novel isolate has a slightly lower optimal growth temperature than the majority of its closest relatives.

Therefore, from the clear genotypic distance, the many physiological similarities and some phenotypical differences, we comply with the phylo-phenetic concept that currently prevails for the description of a new species. Thus, we proposed to assign strain Iri35 $c^{T}$ to a novel species, for which the name Thermococcus camini sp. nov. is proposed.

\section{DESCRIPTION OF THERMOCOCCUS CAMINI SP. NOV.}

Thermococcus camini (ca.mi'ni. L. gen. n. camini of a furnace, referring to the isolation of the type strain from a hydrothermal chimney).

Cells are irregular motile cocci (diameter: $1.1 \pm 0.2 \mu \mathrm{m}$ ). Obligately anaerobic. Growth is observed at temperatures between 50 and $90^{\circ} \mathrm{C}$ (optimum $75-80^{\circ} \mathrm{C}$ ), at $\mathrm{NaCl}$ concentration from $1-5 \%$ (optimum 2\%) and at $\mathrm{pH}$ from 5.0 to 9.0 (optimum 7.0-7.5). Piezophilic growing optimally under $10-30 \mathrm{MPa}$. $\mathrm{S}^{\circ}$ or L-cystine is not required for growth but definitely stimulates growth. Does not use sulphate, thiosulphate, sulphite, polysulphide, nitrate, nitrite or oxygen ( 0.5 , $5,20 \% \mathrm{v} / \mathrm{v}$ ) as electron acceptors. Chemoorganoheterotrophic growth occurs on complex proteinaceous substrates (yeast extract, peptone, tryptone, beef extract) and growth is slightly enhanced by maltose addition. Fermentation products (on peptone, yeast extract and $S^{\circ}$ ) include isovalerate, isobutyrate, acetate, propionate, formate, succinate/or malate, ammonia, thiosulphate, carbon dioxide and hydrogen sulphide.

The type strain Iri35c $c^{\mathrm{T}}$ (=UBOCCM-2026 $6^{\mathrm{T}}=$ DSM $111003^{\mathrm{T}}$ ) was isolated from a deep-sea chimney rock sample collected at a depth of $2300 \mathrm{~m}$ from a hydrothermal chimney at the Rainbow vent field, Mid-Atlantic Ridge. The genomic DNA $\mathrm{G}+\mathrm{C}$ content of the type strain is $54.63 \mathrm{~mol} \%$.

The GenBank/EMBL/DDBJ accession numbers for the 16S rRNA gene and draft genome sequences are MT921160 and LR881183.1, respectively.

\section{Funding information}

This work was supported by the program MERLIN Abyss to K.A, the 'Laboratoire d'Excellence' LabexMER Axis 3 programs ENDIV and CULTIVENT (ANR-10-LABX-1) to K.A., the Sino-French IRP 1211 MicrobSea to K.A. The study was supported by a grant from the French Ministry of Higher Education and Research, to D.C.

\section{Acknowledgements}

The authors thank the co-chiefs, crew and shipboard scientific party of the oceanographic expedition IRIS. We are grateful to Erwan Roussel for his expert advice on ionic chromatography analyses. We thank Phil Oger for providing us with the type strains of the closest species for physiological comparisons. We acknowledge Hilary G. Morrison who did the sequencing. The LABGeM (CEA/Genoscope and CNRS UMR8030), the France Génomique and French Bioinformatics Institute national infrastructures (funded as part of Investissement d'Avenir program managed by Agence Nationale pour la Recherche, contracts ANR-10INBS-09 and ANR-11-INBS-0013) are acknowledged for support within the MicroScope annotation platform.

\section{Author contributions}

Author contributions: Formal analysis, D.C. and K.A.; Funding acquisition, L.M. and K.A.; Investigation, D.C., E.V., N.G., X.P. and K.A.; Supervision, L.M., Z.S. and K.A.; Validation, D.C., E.V., N.G. and K.A.; Writing-original draft, D.C. and K.A.; Writing-review and editing, D.C., L.M., Z.S. and K.A. All authors have read and agreed to the published version of the manuscript.

Conflicts of interest

The authors declare that there are no conflicts of interest.

References

1. Godfroy A, François D, Hartunians J, Moalic Y, Alain K. (in press). Vetriani C and Giovannelli D (eds). In: The Microbiology of Deep-Sea. Physiology, Metabolism and Ecology of Thermophiles from Deep-sea Vents. Springer International;

2. Bertoldo C, Antranikian G. The order Thermococcales. Dworkin M, Falkow S, Rosenberg E, Schleifer KH and Stackerbrandt E (eds). In: The Prokaryotes, Vol. 3. New York: Springer; 2006. pp. 69-81.

3. KozhevnikovaDA, TaranovEA, LebedinskyAV,Bonch-OsmolovskayaEA, Sokolova TG. Hydrogenogenic and sulfidogenic growth of Thermococcus archaea on carbon monoxide and formate. Microbiology 2016:85:400-410.

4. Sokolova TG, Jeanthon C, Kostrikina NA, Chernyh NA, Lebedinsky A, et al. The first evidence of anaerobic $\mathrm{CO}$ oxidation coupled with $\mathrm{H} 2$ production by a hyperthermophilic archaeon isolated from a deepsea hydrothermal vent. Extremophiles 2004;8:317-323.

5. Atomi H, Reeve J. Microbe Profile: Thermococcus kodakarensis: the model hyperthermophilic archaeon. Microbiol 2019:165:1166-1168.

6. Thiel A, Michoud G, Moalic Y, Flament D, Jebbar M. Genetic manipulations of the hyperthermophilic piezophilic archaeon Thermococcus barophilus. Appl Environ Microbiol 2014;80:2299-2306. 
7. Atomi H, Fukui T, Kanai T, Morikawa M, Imanaka T. Description of Thermococcus kodakaraensis sp. nov., a well studied hyperthermophilic archaeon previously reported as Pyrococcus sp. Archaea 2004:1:263-267.

8. González JM, Kato C, Horikoshi K. Thermococcus peptonophilus sp. nov., a fast-growing, extremely thermophilic archaebacterium isolated from deep-sea hydrothermal vents. Arch Microbiol 1995;164:159-164.

9. Zhao W, Zeng X, Xiao X. Thermococcus eurythermalis sp. nov., a conditional piezophilic, hyperthermophilic archaeon with a wide temperature range for growth, isolated from an oilimmersed chimney in the Guaymas Basin. Int J Syst Evol Microbiol 2015;65:30-35.

10. Jolivet E, L'Haridon S, Corre E, Forterre P, Prieur D. Thermococcus gammatolerans sp. nov., a hyperthermophilic archaeon from a deep-sea hydrothermal vent that resists ionizing radiation. Int J Syst Evol Microbiol 2003;53:847-851.

11. Dalmasso C, Oger P, Selva G, Courtine D, L'Haridon S, et al. Thermococcus piezophilus sp. nov., a novel hyperthermophilic and piezophilic archaeon with a broad pressure range for growth, isolated from a deepest hydrothermal vent at the Mid-Cayman Rise. Syst Appl Microbiol 2016:39:440-444.

12. Marteinsson VT, Birrien JL, Reysenbach AL, Vernet M, Marie D, et al. Thermococcus barophilus sp. nov., a new barophilic and hyperthermophilic archaeon isolated under high hydrostatic pressure from a deep-sea hydrothermal vent. Int J Syst Bacteriol 1999:49:351-359.

13. Keller M, Braun FJ, Dirmeier R, Burggraf S, Rachel R, et al. Thermococcus alcaliphilus sp. nov., a new hyperthermophilic archaeum growing on polysulfide at alkaline $\mathrm{pH}$. Arch Microbiol 1995; 164:390-395.

14. Zeng X, Birrien JL, Fouquet $\mathrm{Y}$, Cherkashov G, Jebbar M, et al. Pyrococcus $\mathrm{CH} 1$, an obligate piezophilic hyperthermophile: extending the upper pressure-temperature limits for life. ISME J 2009;3:873-876.

15. Eren AM, Vineis JH, Morrison HG, Sogin ML. A filtering method to generate high quality short reads using illumina paired-end technology. PLOS ONE 2013;8:e66643.

16. Minoche $A E$, Dohm JC, Himmelbauer $H$. Evaluation of genomic high-throughput sequencing data generated on Illumina HiSeq and Genome Analyzer systems. Genome Biol 2011;12:R112.

17. Vallenet D, Calteau A, Dubois M, Amours P, Bazin A, et al. MicroScope: an integrated platform for the annotation and exploration of microbial gene functions through genomic, pangenomic and metabolic comparative analysis. Nucleic Acids Res 2020;48:D589.

18. Kim OS, Cho YJ, Lee K, Yoon SH, Kim M, et al. Introducing EzTaxone: a prokaryotic $16 \mathrm{~S}$ rRNA gene sequence database with phylotypes that represent uncultured species. Int J Syst Evol Microbiol 2012;62:716-721.

19. Katoh K, Standley DM. MAFFT multiple sequence alignment software version 7 improvements in performance and usability. Mol Biol Evol 2013;30:772-780.

20. Criscuolo A, Gribaldo S. BMGE (Block Mapping and Gathering with Entropy): a new software for selection of phylogenetic informative regions from multiple sequence alignments. BMC Evol Biol 2010;10:210

21. Guindon S, Dufayard JF, Lefort V, Anisimova M, Hordijk W, et al. New algorithms and methods to estimate maximum-likelihood phylogenies: assessing the performance of PhyML 3.0. Syst Biol 2010;59:307-321.

22. Lefort V, Longueville J-E, Gascuel O. SMS: Smart Model Selection in PhyML. Mol Biol Evol 2017;34:2422-2424.

23. Letunic I, Bork P. Interactive Tree Of Life (iTOL) v4: recent updates and new developments. Nucleic Acids Res 2019;47:W259.
24. Yoon SH, Ha SM, Lim JM, Kwon SJ, Chun J. A large-scale evaluation of algorithms to calculate average nucleotide identity. Antonie van Leeuwenhoek 2017;110:1281-1286.

25. Richter M, Rosselló-Móra R, Glöckner FO, Peplies J. JSpeciesWS: a web server for prokaryotic species circumscription based on pairwise genome comparison. Bioinformatics 2016;32:929-931.

26. Meier-Kolthoff JP, Auch AF, Klenk H-P, Göker M. Genome sequencebased species delimitation with confidence intervals and improved distance functions. BMC Bioinformatics 2013;14:60.

27. Richter M, Rossello-Mora R. Shifting the genomic gold standard for the prokaryotic species definition. Proc Natl Acad Sci USA 2009:106:19126-19131.

28. Wayne LG, Stackebrandt E, Kandler O, Colwell RR, Krichevsky M, et al. Report of the ad hoc committee on reconciliation of approaches to bacterial systematics. Int J Syst Bacteriol 1987;37:463-464.

29. Rosselló-Móra R, Whitman WB. Dialogue on the nomenclature and classification of prokaryotes. Syst Appl Microbiol 2019:42:5-14.

30. Alain K,Marteinsson VT, Miroshnichenko M,Bonch-OsmolovskayaE, Prieur D, et al. Marinitoga piezophila, sp. nov., a rod-shaped thermopiezophilic bacterium isolated under high hydrostatic pressure from a deep-sea hydrothermal vent. Int J Syst Evol Microbiol 2002:52:1331-1339.

31. Cord-Ruwisch R. A quick method for the determination of dissolved and precipitated sulfides in cultures of sulfate-reducing bacteria. Journal of Microbiological Methods 1985;4:33-36.

32. Miroshnichenko ML, Gongadze GM, Rainey FA, Kostyukova AS, Lysenko AM, et al. Thermococcus gorgonarius sp. nov. and Thermococcus pacificus sp. nov.: heterotrophic extremely thermophilic archaea from New Zealand submarine hot vents. Int J Syst Bacteriol 1998;48:23-29.

33. Kengen SWM, Stams AJM. Formation of l-alanine as a reduced end product in carbohydrate fermentation by the hyperthermophilic archaeon Pyrococcus furiosus. Arch Microbiol 1994;161:168-175.

34. Ward DE, Kengen SW, van Der Oost J, de Vos WM. Purification and characterization of the alanine aminotransferase from the hyperthermophilic archaeon Pyrococcus furiosus and its role in alanine production. J Bacteriol 2000;182:2559-2566.

35. Sakuraba H, Goda S, Ohshima T. Unique sugar metabolism and novel enzymes of hyperthermophilic archaea. Chem Record 2004:3:281-287.

36. Lipscomb GL, Schut GJ, Scott RA, Adams MWW. SurR is a master regulator of the primary electron flow pathways in the order Thermococcales. Mol Microbiol 2017;104:869-881.

37. Topçuoğlu BD, Meydan C, Orellana R, Holden JF. Formate hydrogenlyase and formate secretion ameliorate $\mathrm{H} 2$ inhibition in the hyperthermophilic archaeon Thermococcus paralvinellae. Environ Microbiol 2018;20:949-957.

38. Kuwabara T, Minaba M, Ogi N, Kamekura M. Thermococcus celericrescens sp. nov., a fast-growing and cell-fusing hyperthermophilic archaeon from a deep-sea hydrothermal vent. Int J Syst Evol Microbiol 2007:57:437-443.

39. Grote R, Li L, Tamaoka J, Kato C, Horikoshi K, et al. Thermococcus siculi sp. nov., a novel hyperthermophilic archaeon isolated from a deep-sea hydrothermal vent at the Mid-Okinawa Trough. Extremophiles 1999:3:55-62.

40. Hensley SA, Jung JH, Park CS, Holden JF. Thermococcus paralvinellae sp. nov. and Thermococcus cleftensis sp. nov. of hyperthermophilic heterotrophs from deep-sea hydrothermal vents. Int J Syst Evol Microbiol 2014:64:3655-3659.

41. Holden JF, Takai K, Summit M, Bolton S, Zyskowski J, et al. Diversity among three novel groups of hyperthermophilic deep-sea Thermococcus species from three sites in the northeastern Pacific Ocean. FEMS Microbiol Ecol 2001;36:51-60. 